\title{
THE NOVEMBER MEETING IN NORMAN
}

The four hundred seventy-fifth meeting of the American Mathematical Society, was held at the University of Oklahoma on Friday and Saturday, November 23-24, 1951. There were about 50 registrations including the following 29 members of the Society:

R. V. Andree, Arthur Bernhart, J. C. Brixey, Y. W. Chen, L. A. Colquitt, N. A. Court, G. M. Ewing, Casper Goffman, I. E. Glover, A. A. Grau, E. V. Greer, O. H. Hamilton, J. O. Hassler, W. N. Huff, L. W. Johnson, A. E. Labarre, G. Q. LaFon, H. W. Linscheid, Dora McFarland, B. L. Mackin, W. C. Orthwein, C. J. Pipes, E. H. Rothe, G. W. Smith, R. G. Smith, O. S. Spears, C. E. Springer, E. B. Stouffer, J. W. T. Youngs.

By invitation of the Committee to Select Hour Speakers for Western Sectional Meetings, Professor E. H. Rothe of the University of Michigan delivered an address entitled Gradient mappings at 2:00 P.M. on Friday. Dean E. B. Stouffer presided at the session.

Sessions for the presentation of contributed papers were held at 3:30 P.M. on Friday and 10:00 A.M. on Saturday. Presiding officers at these sessions were Professors C. E. Springer and O. H. Hamilton.

The Society was entertained by President and Mrs. Cross of the University of Oklahoma at a tea in their residence on Friday afternoon. That same evening there was a dinner in the Union for the Society and its guests.

The meeting was characterized by a pleasant air of informality, together with active participation in the discussions following the papers by a large percentage of the audience.

The Society is indebted to the Committee on Local Arrangements for a well planned meeting, and for the obvious pains which were taken to look after the physical comfort of the visitors.

\section{Algebra and Theory of Numbers}

106. D. W. Dubois: Partly ordered fields. Preliminary report.

A commutative field $K$ is called a partly ordered field if a relation " $x$ is positive" (abbreviated " $x>0$ ") is defined for at least one $x \in K$ such that if $a$ and $b$ are positive, so are $a+b, a b$, and $a b^{-1}$. One defines $a<0, x>y, x<y$ as usual. One shows that every partly ordered field is a partly ordered set containing the rational field in its natural order and that every field of characteristic zero can be partly ordered. The partial order of $K$ is said to be Archimedean if for every $x \in K$ there is an integer $n \in K$ with $n>x$. Examples are given of both Archimedean and non-Archimedean partly ordered fields which are not simply ordered by the partial orderings. The following results are proved for an arbitrary partly ordered field $K:$ (1) $K$ contains a subfield $D$ already ordered by the partial order of $K$, and $D$ contains every other such subfield. $D$ con- 
sists of Dedekind cuts on the rational subfield. (2) There is a real-valued norm $\mu(x)$ on $K$; the completion of $K$ by fundamental sequences yields a real Banach algebra. (3) If $K$ is an algebraic number field, $K$ can be Archimedean partly ordered if and only if $K$ is formally real. (Received October 9, 1951.)

\section{AnALysis}

107. Y. W. Chen: On hodograph transformations of partial differential equations with three independent variables.

A system of three quasi-linear partial differential equations (1): $A_{i j} \cdot \partial u_{i} / \partial x_{j}=0$, $B_{i j} \cdot \partial u_{i} / \partial x_{j}=0$, and $C_{i j} \cdot \partial u_{i} / \partial x_{j}=0$ is considered for three functions $u_{i}\left(x_{1}, x_{2}, x_{3}\right)$, $i=1,2,3$. The coefficients $A_{i j}, B_{i j}$, and $C_{i j}$ are functions of $(u)$ and do not depend on $(x)$. By means of the hodograph transformations nine functions $\zeta_{i j}\left(u_{1}, u_{2}, u_{8}\right)$ are introduced with $u_{i}$ as independent variables. They satisfy the following system (2) of nine equations: (2.1) $A_{i j}(u) \cdot \zeta_{i j}(u)=0, B_{i j}(u) \cdot \zeta_{i j}(u)=0, C_{i j}(u) \zeta_{i j}(u)=0$, (2.2) $\partial \zeta_{1 j} / \partial u_{1}+\partial \zeta_{2 i} / \partial u_{2}+\partial \zeta_{3 j} / \partial u_{3}=0, j=1,2,3,(2.3) \xi_{i 3}\left(\partial \xi_{i 1} / \partial u_{2}-\partial \xi_{i 2} / \partial u_{1}\right)+\xi_{i 1}\left(\partial \xi_{i 2} / \partial u_{3}\right.$ $\left.-\partial \xi_{i 3} / \partial u_{2}\right)+\xi_{i 2}\left(\partial \xi_{i 3} / \partial u_{1}-\partial \xi_{i 1} / \partial u_{3}\right)=0, i=1,2,3$, where $\xi_{\alpha j}=\left(\zeta_{h \beta} \zeta_{l \gamma}-\zeta_{k \gamma} \zeta_{l \beta}\right)$ and the indices $(\alpha, \beta, \gamma),(j, k, l)$ are cyclic permutations of $(1,2,3)$. It is shown that systems (1) and (2) are equivalent for initial values problems. System (2) is also applied to study series expansions of three-dimensional steady subsonic flows. (Received October 11, 1951.)

\section{E. V. Greer: Certain relations between functions.}

A real function $g(x)$ on $[0,1]$ will be called related to $f(x)$ in the Saks-Sierpinski sense if for every $\epsilon>0$ the inequality $|f(x)-g(x)|<\epsilon$ holds on a set of exterior measure 1. It will be called related to $f(x)$ in the Blumberg sense if $f(x)$ fully approaches $g(x)$ almost everywhere. It is shown that a measurable $g(x)$ is related to $f(x)$ in the SaksSierpinski sense if and only if it is related to $f(x)$ in the Blumberg sense. This fact is used to prove the following generalization of the fundamental theorem of the integral calculus: If $f(x)$ is a bounded, single-valued function defined on $[0,1]$, then the derivative of the upper Lebesgue integral of $f(x)$ is related to $f(x)$ in the SaksSierpinski sense. In connection with these ideas, the following analogue of a theorem of Saks and Sierpinski is proved for planar transformations: If $f(z)$ is an arbitrary single-valued transformation of the closed unit square into the euclidean plane, then there is a single-valued transformation $g(z)$ of Baire class 2 at most which is related to $f(z)$ in the Saks-Sierpinski sense. (Received October 2,1951.)

109. W. N. Huff and. E. D. Rainville: On the Sheffer A-type of polynomials generated by $\phi(t) f(x t)$.

The Sheffer A-type of the polynomial set $\left\{y_{n}(x)\right\}$ defined by $f(x t) \phi(t)=\sum_{n=0}^{\infty} y_{n}(x) t^{n}$ was discussed in a previous paper by Huff. The main result of the present paper is a necessary and sufficient condition that $\left\{y_{n}(x)\right\}$ be of Sheffer A-type $k$ : that $f(x t)$ be a hypergeometric function with no numerator parameters, with $k$ denominator parameters, and with argument a constant multiple of $x t$. (Received September 24, 1951.)

110t. T. S. Motzkin and W. R. Wasow: On the approximate solution of linear elliptic differential equations by difference equations with positive coefficients. 
Let $L[u] \equiv \sum_{i, k=1}^{n} \beta_{i k}(x) \partial^{2} u / \partial x_{i} \partial x_{k}+\sum_{j=1}^{n} \alpha_{j}(x) \partial u / \partial x_{j}-c(x) u$ be an elliptic differential expression. Here $x$ denotes the vector with components $x_{1}, x_{2}, \cdots, x_{n}$. The finite difference expression $L_{h}[u] \equiv \sum_{s=1}^{N} c_{s}(x, h) u\left(x_{1}+m_{1 s} h, \cdots, x_{n}+m_{n s} h\right)-u(x)$ in a square lattice of mesh length $h$ is called formally analogous to $L[u]$ if the beginning of Taylor's expansion of $L_{h}[u]$ about the point $x$ is proportional to $L[u]$. The study of the relation between the solutions of boundary value problems in a bounded domain $B$ belonging to formally analogous differential and difference expressions is greatly simplified if all $c_{s}(x, h)$ are non-negative for $x$ in $B$. It is shown that, for $n>1$, this condition cannot be satisfied if the set of integers $m_{i s}$ is to be the same for all elliptic differential expressions. This is true even for the subclass of expressions $L[u]$ whose coefficients are bounded in absolute value by a given number $M$, for all $x$ in $B$. However, if the further restriction is imposed that Det $\left\{\beta_{i k}(x)\right\}$ is to exceed in $B$ a positive, otherwise arbitrary, number, then a set of integers $m_{i s}$, independent of $L[u]$, exists for which the $c_{s}(x, h)$ can be chosen positive in $B$. (Received August 17, 1951.)

\section{C. J. Pipes: Generalizations of a theorem of Sierpinski and Zyg-} mund on continuous functions.

Consider an abstract set property $\pi$ defined by the following postulates. $\pi$ is a set property such that: (1) every subset, including the empty set, of a set that has the property also has the property, (2) the union of a finite or denumerable number of sets that have the property also has the property, (3) every set consisting of a single point has the property, and (4) the continuum does not have the property. Then, for any set property $\pi$, if continuity is generalized to $\pi$-continuity by considering as negligible those sets that satisfy the property, the following result may be obtained: there is a real function that is not $\pi$-continuous on any set, relative to the set, not satisfying the property $\pi$. An analogous result may be obtained if continuity is generalized by considering as negligible all sets of exterior metric density zero at a point. (Received October 10, 1951.)

\section{Applied Mathematics}

\section{2t. Abolghassem Ghaffari: A new approximation method for com-} pressible subsonic flow.

The following investigations are an attempt to find an approximation to the velocity potential $\phi$ and stream function $\psi$ for compressible flow. If $\tau_{1}$ is the value of $\tau=w^{2} / w_{m}^{2}$ (where $w$ is the fluid speed and $w_{m}$ is the maximum fluid speed) in the main stream, the Mach number in the main stream is given by $M_{1}=\tau_{1} /\left(1-\tau_{1}\right)$. Hence the effects of compressibility will diminish as $M_{1}$ and $\tau_{1}$ tend to zero. Therefore, to pass from a compressible flow to a corresponding incompressible flow, we put $\tau=q^{2} \tau_{1}$ (where $q$ is the velocity in incompressible flow) in the expressions of $\phi$ and $\psi$, and then let $\tau_{1}$ approach zero. The simplified form of hodograph equations are (1) $P \partial \psi / \partial \tau$ $=\partial \phi / \partial \theta, Q \partial \phi / \partial \tau=-\partial \psi / \partial \theta$, where $P=2 \tau(1-\tau)^{-\beta}, Q=2 \tau(1-\tau)^{\beta+1}[1-(2 \beta+1) \tau]^{-1}$; $\beta=(\gamma-1)^{-1} ; \gamma$ is adiabatic index. The corresponding limiting form of (1) is (2) $q \partial \phi / \partial q=-\partial \psi / \partial \theta, q \partial \psi / \partial q=\partial \phi / \partial \theta$. One of the elementary solutions of (2) is (3) $\phi=C_{m} q^{m} \cos \left(m \theta+\epsilon_{m}\right), \psi=-C_{m} q^{m} \sin \left(m \theta+\epsilon_{m}\right)$, where $C_{m}, \epsilon_{m}$ are constants and $m$ is any nonzero number. It is found that the corresponding elementary solutions of (3) for $m>0$ are (4) $\psi=-C_{m} \psi_{m}(\tau) / \psi_{m}\left(\tau_{1}\right) \sin \left(m \theta+\epsilon_{m}\right), \phi=(P / m) \partial \psi / \partial \tau$; and for 
$m=-n<0$ are (5) $\psi=-C_{-n} \psi_{-n}(\tau) / \psi_{-n}\left(\tau_{1}\right) \sin \left(-n \theta+\epsilon_{-n}\right), \phi=-(P / n) \partial \psi / \partial \tau$, where $\psi_{-n}(\tau)=\left[\tau^{-n / 2}-K \tau^{n / 2} \tau_{s}^{-n}\right][1+O(\tau)]$, and $K$ is independent of $\tau, \tau_{s}$ being the value of $\tau$ at the sonic speed. It is shown that in the subsonic range when $m>0$ the two functions $\psi_{m}$ and $\phi_{m}$ are both positive monotonically increasing functions of $\tau$. (Received September 20, 1951.)

\section{3t. Abolghassem Ghaffari: Asymptotic behaviour of compressible} subsonic flow.

Let $\psi$ and $\phi$ denote the stream function and the velocity potential of a compressible flow and let $\tau=w^{2} / w_{m}^{2}$ and $\theta$ be the hodograph variables, where $w$ is the fluid speed and $w_{m}$ is the maximum fluid speed. Chaplygin's formal solution (1) $\psi=B \theta$ $+\sum_{m=1}^{\infty} A_{m} \psi_{m}(\tau) \sin \left(m \theta+\epsilon_{m}\right), \phi=-B \phi_{0}(\tau)-\sum_{m=1}^{\infty} A_{m} \phi_{m} \cos \left(m \theta+\epsilon_{m}\right)$ are exact, but they are quite unsuitable for numerical calculations. The author endeavors to determine the asymptotic expansions of $\psi$ and $\phi$ for large values of $m$. Considering the simplified form of hodograph equations and setting $\psi_{m}=\exp \left(\int f_{m} d \tau\right)$ where $f_{m}(\tau)$ is an unknown function, one finds that (2) $\psi_{m} \sim h(\tau) \exp \left[m g(\tau)-m g\left(\tau_{1}\right)\right]$, $\phi_{m} \sim h^{-1}(\tau)$ exp $\left[m g(\tau)-m g\left(\tau_{1}\right)\right]$, where $h(\tau)=(1-\tau)^{(2 \beta+1) / 4}[1-(2 \beta+1) \tau]^{-1 / 4}, g(\tau)$ $=\int_{\tau_{1}}^{\tau}(2 \tau)^{-1}(1-\tau)^{-1 / 2}[1-(2 \beta+1) \tau]^{1 / 2} d \tau, \quad \tau_{1}$ being the value of $\tau$ in the main stream. Taking into account (2), the formal solutions (1) become (3) $\psi$ $\sim h(\tau) \sum_{1}^{\infty} A_{m} \exp \left[m g(\tau)-m g\left(\tau_{1}\right)\right] \sin \left(m \theta+\epsilon_{m}\right), \phi r-h^{-1}(\tau) \sum_{1}^{\infty} A_{m} \exp [m g(\tau)$ $\left.-m g\left(\tau_{1}\right)\right] \cos \left(m \theta+\epsilon_{m}\right)$. Now let $w_{m} \rightarrow \infty$, then $g(\tau)-g\left(\tau_{1}\right) \rightarrow 1 / 2 \log \tau / \tau_{1}$; therefore, the final expressions of $\psi$ and $\phi$ for small $\tau$ approach (4) $\psi \sim \sum_{1}^{\infty} A_{m}\left(w / w_{1}\right)^{m} \sin \left(m \theta+\epsilon_{m}\right)$, $\phi \sim-\sum_{1}^{\infty} A_{m}\left(w / w_{1}\right)^{m} \cos \left(m \theta+\epsilon_{m}\right)$, where $w_{1}$ is the fluid speed in the main stream. As we are dealing with subsonic flow in which $0 \leqq \tau \leqq \tau_{1} \leqq \tau_{s}$, the series (3) and (4) are absolutely and uniformly convergent. (Received September 20,1951.)

\section{GEOMETRY}

\section{N. A. Court: Isogonal points for a tetrahedron.}

Let $(M),\left(M^{\prime}\right)$ be the pedal tetrahedrons for tetrahedron $(T)=A B C D$ of the points $M, M^{\prime}$ isogonal for $(T),(L)$ the common pedal sphere of $M, M^{\prime}$ for $(T)$, and $(A)$, $(B),(C),(D)$, the spheres orthogonal to $(L)$ and having for centers the vertices of $(T)$. The following are some of the results obtained. a. The polar planes of $M$ for the four spheres $(A), \cdots,(D)$ coincide with the faces of the tetrahedron $\left(M^{\prime}\right) ; \mathrm{b}$. The lines joining $M$ to the vertices of $(T)$ meet the respective faces of $(M)$ in four points forming a tetrahedron which is affine to the analogous tetrahedron obtained for the point $M^{\prime}$; c. The projections of the vertices of the two tetrahedrons $(M),\left(M^{\prime}\right)$ upon the line $M M^{\prime}$ are two tetrads of points having the same biratio (that is, anharmonic ratio). The value of this biratio is equal to the biratio of the pencil of planes $M M^{\prime}(A B C D)$. (Received October 8, 1951.)

\section{TOPOLOGY}

\section{Arthur Bernhart: Seven-rings in minimal five-color maps.}

The simultaneous system of linear homogeneous equations in twice 91 unknowns, together with inequalities derived from the minimal nature of the map, are solved. Over 150 solutions have been obtained. Unless there are topological conditions stronger than these colorability requirements, an indirect proof of the 4-color theorem seems unlikely. (Received October 9, 1951.) 
116. G. M. Ewing and W. R. Utz: Continuous solutions of the functional equation $f^{n}(x)=f(x)$.

Let $f^{n}(x)$ denote $f$ iterated $n$ times. The authors find all continuous real functions defined on the real axis such that for an integer $n \geqq 2$ the stated equation is satisfied. (Received November 23, 1951.)

J. W. T. Youngs, Associate Secretary 